\title{
The impact of improved Stark-broadening widths on the modeling of double-ionized chromium lines in hot stars
}

\author{
A. Chougule ${ }^{1,2}$, N. Przybilla ${ }^{1}$, M.S. Dimitrijević ${ }^{2}$ \\ and V. Schaffenroth ${ }^{3}$ \\ ${ }^{1}$ Institut fr Astro- und Teilchenphysik, Universitt Innsbruck, \\ Technikerstr. 25/8, A-6020 Innsbruck, Austria \\ (E-mail: abhishek.rajeshchougule@gmail.com) \\ 2 Astronomical Observatory, Volgina 7, 11060 Belgrade 38, Serbia \\ 3 Institute for Physics and Astronomy, University of Potsdam, \\ Karl-Liebknecht-Str. 24/25, \\ 14476 Potsdam, Germany,
}

Received: July 31, 2019; Accepted: November 6, 2019

\begin{abstract}
Stellar atmosphere modeling and chemical abundance determinations require the knowledge of spectral line shapes. Spectral lines of chromium in various ionization stages are common in stellar spectra but detailed data on Stark broadening for them is scarce. Recently we reported on the first calculations of Stark widths for several $4 s-4 p$ transitions of double-ionized chromium, employing the Modified Semi-Empirical approach (MSE). In this work we present applications of the data to spectrum synthesis of Cr III lines in the ultraviolet region of hot stars. The ATLAs9 model atmosphere code and the line-formation code SURFACE were used with the assumption of local thermodynamic equilibrium. The improvements of adopting the MSE broadening tables instead of approximate Stark broadening coefficients are investigated for a total of 56 Cr III lines visible in HST/STIS spectra of the B3 subgiant star Iota Herculis and the subdwarf B-star Feige 66.
\end{abstract}

Key words: Line: profiles - Stars: abundances

\section{Introduction}

Most of our knowledge of the physical conditions of astrophysical systems relies on the observations of electromagnetic radiation emitted by them. For example, a stellar spectrum can reveal many properties of stars, such as their chemical composition, temperature, density, distance, mass, luminosity, and radial motion using Doppler shift measurements. The stellar spectral lines are not infinitely sharp but have a finite width due to various physical processes. Each of these processes introduces a characteristic shape that can be expressed in the form of a line profile function $\phi(\nu)$ where the parameters of a normalized line profile $\left(\int \phi(\nu) d \nu=1\right)$ are the central frequency $\nu_{0}$, the Full Width at Half Maximum 
(FWHM) and the profile value at the central frequency $\phi\left(\nu_{0}\right)$. The knowledge of spectral line shapes is required for stellar atmosphere modeling and chemical abundance determinations. In this work, we focus on the study of line broadening in stars due to collisions with neighboring charged particles, i.e., Stark broadening, in particular for double-ionized chromium Cr III.

\subsection{Stark broadening}

In plasma, collisional interactions between emitters/absorbers - atoms and ions, and charged particles - free electrons and ions, results in the broadening of spectral lines, called Stark broadening. The corresponding line profile, due to the application of the impact approximation - the interactions are separated in time -, and the isolated line approximation - neighboring energy levels do not overlap -, (Baranger, 1958), results in a Lorentzian profile generally defined by the corresponding FWHM and shift. The FWHM governs the shape of the line while shift (which is usually less than the width), determines the position relative to the central wavelength of the line, and it is not so important for studying the influence of Stark broadening in stellar spectra and has been neglected in our work. The isolated line approximation holds true for most of the spectral lines originating from stellar atmosphere (Griem \& Ralchenko, 2000) as well as for spectral lines considered in this work, for electron densities of interest.

In order to produce a synthetic stellar spectrum that can be compared to observations, line broadening mechanisms other than Stark broadening also need to be taken into consideration, like the natural line width and Doppler broadening. The resulting line profile is a convolution of the individual profile functions, known as Voigt profile. Hence, any change in the FWHM due to Stark broadening is reflected in the resulting synthetic stellar spectrum. It is worth noting that the FWHM is proportional to the damping constant describing a spectral line. In fact, damping constants have been widely used for stellar spectral synthesis. In our investigation, for the calculation of synthetic spectra based on approximate Stark broadening data, the damping constants obtained using the methods described in Sec. 2.1 were used. On the other hand, for the calculation of synthetic spectra based on the broadening tables, the MSE calculated FWHMs were included into the input file.

\section{Method}

We study and compare the effects of improved Stark broadening tables for Cr III lines (Dimitrijević \& Chougule, 2018) and approximate Stark broadening constants as described in Castelli (2005) on the calculation of synthetic stellar spectra. We also compare these spectra with observations, for the B3 subgiant star Iota Herculis and the subdwarf B-star Feige 66. 


\subsection{Approximate Stark broadening data}

As reported in Castelli (2005), the Stark damping constant used for line computation is a fit by Peytremann (1972) to the detailed calculations by SahalBréchot \& Segre (1971):

$$
\gamma_{\text {Stark }}=10^{-8} n^{*^{5}} N_{\mathrm{e}}\left(\mathrm{s}^{-1}\right)
$$

Here, $n^{*}=\left[\frac{E_{\mathrm{H}} Z_{\text {eff }}^{2}}{\chi_{\mathrm{ion}}-\chi_{\mathrm{k}}}\right]^{\frac{1}{2}}$ and is known as the effective quantum number of the state $k$ (upper state). The ionization energy of hydrogen is given by $E_{\mathrm{H}}=$ $13.6 \mathrm{eV}, Z_{\text {eff }}$ is the effective charge (e.g., 1 for Cr I, 2 for Cr II, etc.), $\chi_{\text {ion }}$ is the ionization potential, and $\chi_{\text {upper }}$ is the excitation potential of the upper level. If the upper level is above the ionization potential, because it ionizes to an excited level of the parent, $n^{*}$ is set to 5 . The damping constant $\gamma_{\text {Stark }}$ is related to the half-half width $(W)$ listed by Griem \& Peach (1975) via the expression:

$$
\frac{\gamma_{\text {stark }}}{N_{\mathrm{e}}}=\frac{2 \pi c(2 W)}{\lambda_{0}^{2}} \frac{1}{N_{\mathrm{e}}}
$$

where $N_{\mathrm{e}}=10^{16} \mathrm{~cm}^{-3}$ for neutral atoms and $N_{\mathrm{e}}=10^{17} \mathrm{~cm}^{-3}$ for ions. In the Kuruczs line lists, the $\mathrm{W}$ values from Griem \& Peach (1975) are usually for $T=10,000 \mathrm{~K}$.

\subsection{Improved Stark broadening data}

The improved Stark broadening data of Dimitrijević \& Chougule (2018) were calculated using the Modified Semi-empirical (MSE) approach (Dimitrijevic \& Konjević, 1980; Dimitrijević \& Kršljanin, 1986; Dimitrijević \& Popović, 2001). The accuracy of the MSE approach is estimated to be about $\pm 50 \%$ in general (Dimitrijević \& Popović, 2001) and it has been tested on numerous occasions (Dimitrijević, 1990). Even for emitters with very complex spectra (e.g. Xe II and $\mathrm{Kr}$ II) good agreement of the MSE approach with experimental measurements (in the range $\pm 30 \%$ ) can be achieved (Popović \& Dimitrijević, 1996, 1998).

\subsection{Stellar spectral synthesis}

A hybrid non local thermodynamic equilibrium (non-LTE) approach was used for the model calculations as presented by Nieva \& Przybilla (2007) for Btype main-sequence stars and Przybilla et al. (2006) for sdB stars. The model stellar atmospheres were assumed to be in LTE and were computed with the ATLAs9 code (Kurucz, 1993). Subsequently, deviations from LTE (non-LTE) were considered in the line-formation computations with DETAIL and SURFACE (Giddings 1981; Butler \& Giddings 1985; recently extended and updated by K. Butler). The non-LTE calculations for hydrogen and helium were based on model atoms of Przybilla \& Butler (2004) and Przybilla (2005), respectively. The 
line formation calculations for chromium were performed assuming LTE because the lack of a proper non-LTE model atom, based on input data described by Schaffenroth (2015). Both, calculations with the approximate Stark broadening coefficients and with the MSE tables were performed for the two test stars, with atmospheric parameters adopted from Nieva \& Przybilla (2012) and O'Toole \& Heber (2006), respectively. Effective temperature $T_{\text {eff }}$, (logarithmic) surface gravities $\log (g)$ and logarithmic chromium abundances relative to hydrogen are summarised in Tab. 1.

Table 1. Properties of the stars under consideration.

\begin{tabular}{llll}
\hline \hline Star & $\log (g)$ & $T_{\text {eff }}$ & Cr abundance \\
\hline Feige 66 & 5.83 & $34,500 \mathrm{~K}$ & -4.70 \\
Iota Herculis & 3.80 & $17,500 \mathrm{~K}$ & -6.40 \\
\hline \hline
\end{tabular}

\subsection{Comparison with observations}

The synthetic spectra were compared to observations of Cr III lines in the UV spectral range. Data obtained with the Space Telescope Imaging Spectrograph (STIS) installed on the Hubble Space Telescope (HST) were downloaded from the Mikulski Archive for Space Telescopes. Both the spectra were observed employing the NUV-MAMA detector and the E230M grating, providing a resolving power of 30,000 in the wavelength range $1605 \AA$ to $3100 \AA$, at moderate signalto-noise ratios.

\section{Results}

The MSE Stark broadening tables included for stellar spectral synthesis correspond to a perturber density of $10^{17} \mathrm{~cm}^{-3}$ and temperatures from $5000 \mathrm{~K}$ up to $80,000 \mathrm{~K}$. A comparison of $56 \mathrm{Cr}$ III synthetic spectral lines based on approximate Stark broadening data with those based on MSE broadening tables was done in the stars Feige 66 and Iota Herculis. As the influence of Stark broadening is seen in the wings of the spectral lines, strong lines are expected to show this effect in their line profiles. For the star Feige 66, the difference in line shapes was quite prominent for almost all the Cr III spectral lines under investigation (see Fig. 1) because the high-density atmosphere pronounces the differences. On the other hand, the difference in line shapes was very small in case of the star Iota Herculis (see Fig. 2) due to it's lower-density subgiant atmosphere. Some of the basic properties of the two stars are as summarized in Tab. 1.

In order to compare with the observation (Fig. 3 and Fig. 4), the theoretical spectra were convolved with the rotational, macroturbulent, and the instrumental profiles. Due to low signal-to-noise ratio of the observed spectra and line 


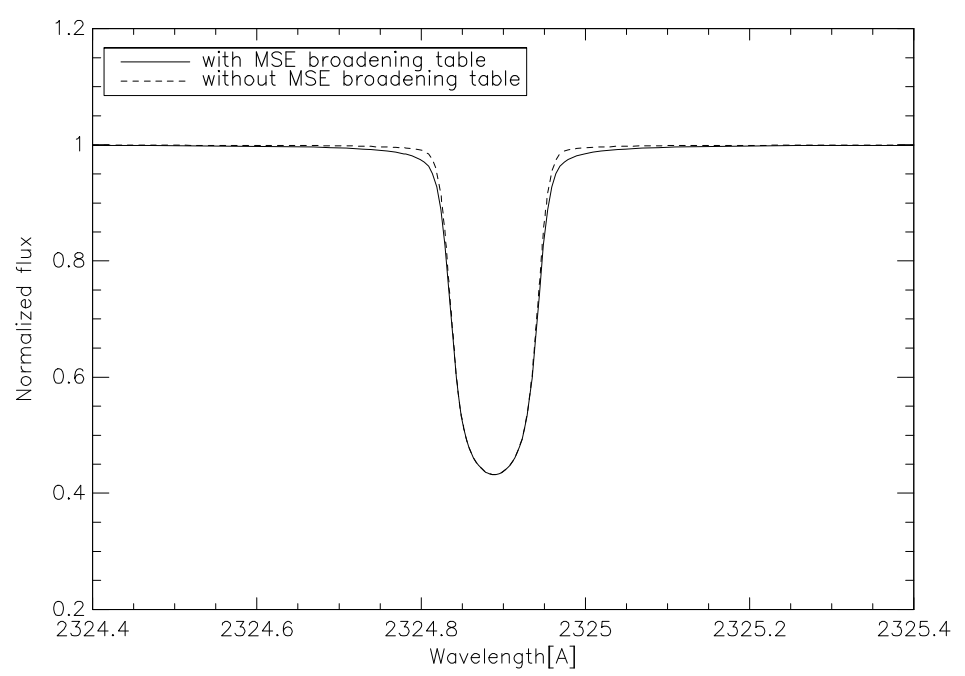

Figure 1. Comparison of the Cr III synthetic spectral lines corresponding to the transition $3 \mathrm{~d}^{3}\left({ }^{4} \mathrm{~F}\right) 4 \mathrm{~s}^{3} \mathrm{~F}-3 \mathrm{~d}^{3}\left({ }^{4} \mathrm{~F}\right) 4 \mathrm{p}^{3} \mathrm{G}^{o}, 2324.889 \AA$ in Feige 66 . The difference in the line profiles can be seen clearly in the wings.

blends, only a few lines were found suitable for comparison with the synthetic spectra.

\section{Conclusions}

We have shown that the strong Cr III lines can react sensitively to the choice of Stark broadening parameters, depending on the perturber densities. Use of the MSE tables yields significantly broader line profiles in the case of the highdensity sdB star Feige 66, while the differences are negligible for the subgiant Iota Herculis. The effects on the abundance determination still need to be quantified, but can already be qualified as being significant. The situation found here is therefore similar to the case of the star HD 133792, for which Dimitrijević et al. (2007) have investigated the impact of Stark broadening data for Cr II lines computed using the semiclassical perturbation approach on the analysis of a high signal-to-noise specctrum. There, the improved data were found to be important, in particular for the interpretation of the chromium abundance stratification. 


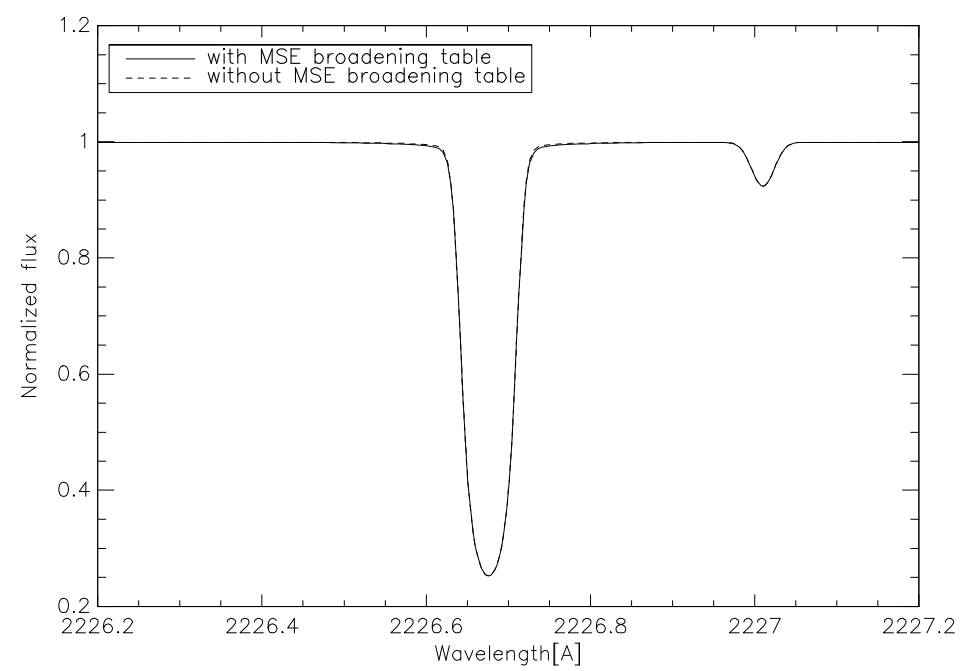

Figure 2. Comparison of the Cr III synthetic spectral lines corresponding to the transition $3 \mathrm{~d}^{3}\left({ }^{4} \mathrm{~F}\right) 4 \mathrm{~s}^{5} \mathrm{~F}-3 \mathrm{~d}^{3}\left({ }^{4} \mathrm{~F}\right) 4 \mathrm{p}^{5} \mathrm{G}^{o}, 2226.676 \AA$ in Iota Herculis. The difference in the line profiles is very small.

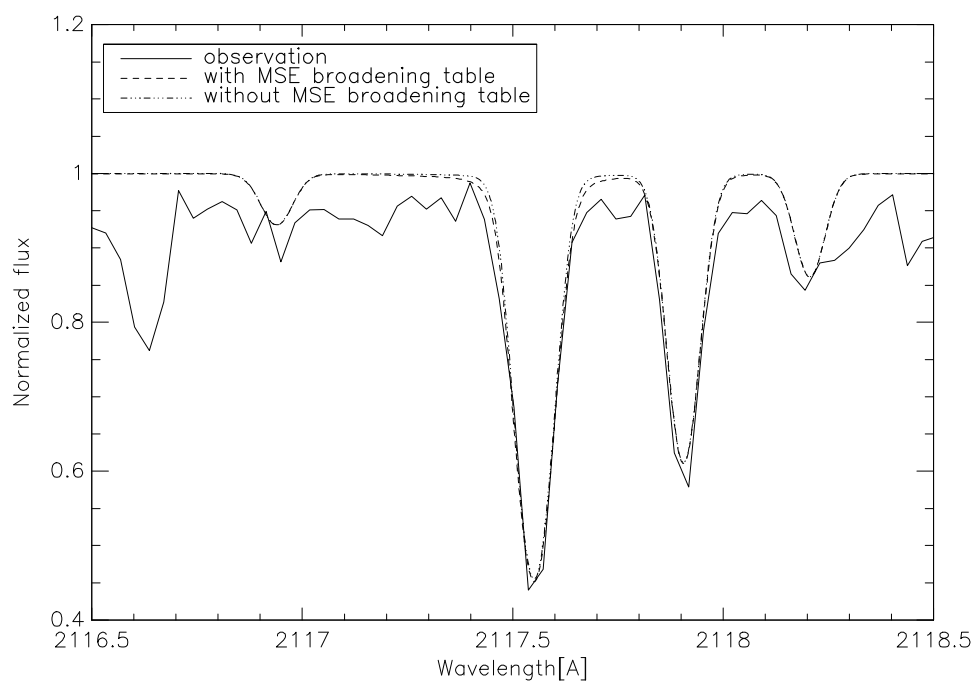

Figure 3. Comparison of the Cr III synthetic spectral lines corresponding to the transition $3 \mathrm{~d}^{3}\left({ }^{4} \mathrm{~F}\right) 4 \mathrm{~s}^{5} \mathrm{~F}-3 \mathrm{~d}^{3}\left({ }^{4} \mathrm{~F}\right) 4 \mathrm{p}^{5} \mathrm{~F}^{o}, 2117.5 \AA$ with observation in Feige 66. 


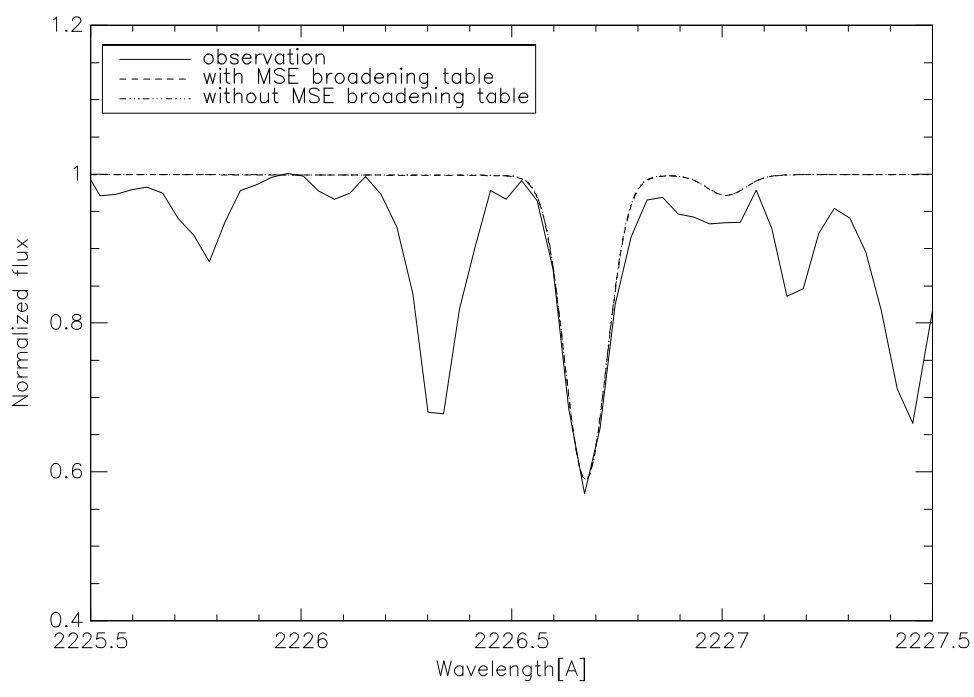

Figure 4. Comparison of the Cr III synthetic spectral lines corresponding to the transition $3 \mathrm{~d}^{3}\left({ }^{4} \mathrm{~F}\right) 4 \mathrm{~s}^{5} \mathrm{~F}-3 \mathrm{~d}^{3}\left({ }^{4} \mathrm{~F}\right) 4 \mathrm{p}^{5} \mathrm{G}^{o}, 2226.676 \AA$ with observation in Iota Herculis.

Acknowledgements. A.C. would like to thank the organizers of the $12^{\text {th }}$ Serbian Conference on Spectral Line Shapes in Astrophysics for financial support and the University of Innsbruck for granting a doctoral scholarship from the Promotion of Young Researchers Program. V.S. acknowledges funding by the Deutsches Zentrum fr Luft- und Raumfahrt, grant 50 OR 1110, under which, part of the work was conducted.

\section{References}

Baranger, M., General Impact Theory of Pressure Broadening. 1958, Physical Review, 112, 855, DOI: 10.1103/PhysRev.112.855

Butler, K. \& Giddings, J. R. 1985, in Newsletter of Analysis of Astronomical Spectra, No. 9 (Univ. London)

Castelli, F., Kurucz's WIDTH code and INPWIDTH. 2005, Memorie della Societa Astronomica Italiana Supplementi, 8, 44

Dimitrijević, M. \& Chougule, A., Stark Broadening of Cr III Spectral Lines: DO White Dwarfs. 2018, Atoms, 6, 15, DOI: 10.3390/atoms6020015

Dimitrijević, M. S., Accuracy of line broadening data. 1990, in Accuracy of Element Abundances from Stellar Atmospheres, ed. R. Wehrse, Lecture Notes in Physics 356 (Springer Verlag), 31, 44

Dimitrijević, M. S. \& Konjević, N., Stark widths of doubly- and triply-ionized atom lines. 1980, J. Quant. Spectrosc. Radiat. Transf., 24, 451, DOI: 10.1016/00224073(80)90014-X 
Dimitrijević, M. S. \& Kršljanin, V., Electron-impact shifts of ion lines: modified semiempirical approach. 1986, Astron. Astrophys., 165, 269

Dimitrijević, M. S. \& Popović, L. Č., Modified Semiempirical Method. 2001, Journal of Applied Spectroscopy, 68, 893

Dimitrijević, M. S., Ryabchikova, T., Simić, Z., Popović, L. Č., \& Dačić, M., The influence of Stark broadening on Cr II spectral line shapes in stellar atmospheres. 2007, Astron. Astrophys., Suppl. Ser., 469, 681, DOI: 10.1051/0004-6361:20077054

Giddings, J. R. 1981, PhD. Thesis, University of London

Griem, H. R. \& Peach, G., Spectral Line Broadening by Plasmas. 1975, Physics Today, 28, 61, DOI: $10.1063 / 1.3068826$

Griem, H. R. \& Ralchenko, Y. V., Electron collisional broadening of isolated lines from multiply-ionized atoms. 2000, J. Quant. Spectrosc. Radiat. Transf., 65, 287, DOI: 10.1016/S0022-4073(99)00074-6

Kurucz, R. 1993, CD-ROM No. 13 (Cambridge, Mass: SAO)

Nieva, M. F. \& Przybilla, N., Hydrogen and helium line formation in OB dwarfs and giants. A hybrid non-LTE approach. 2007, Astron. Astrophys., 467, 295, DOI: 10.1051/0004-6361:20065757

Nieva, M. F. \& Przybilla, N., Present-day cosmic abundances. A comprehensive study of nearby early B-type stars and implications for stellar and Galactic evolution and interstellar dust models. 2012, Astron. Astrophys., 539, A143, DOI: 10.1051/0004$6361 / 201118158$

O'Toole, S. J. \& Heber, U., Abundance studies of sdB stars using UV echelle HST/STIS spectroscopy. 2006, Astron. Astrophys., 452, 579, DOI: 10.1051/00046361:20053948

Peytremann, E., Theoretical Effect of Various Broadening Parameters on Ultraviolet Line Profiles. 1972, Astron. Astrophys., 17, 76

Popović, L. Č. \& Dimitrijević, M. S., Stark broadening of XeII lines. 1996, Astron. Astrophys., Suppl., 116, 359

Popović, L. Č. \& Dimitrijević, M. S., Stark broadening parameters for Kr II lines from 5s-5p transitions. 1998, Astron. Astrophys., Suppl., 127, 295, DOI: 10.1051/aas:1998351

Przybilla, N., Non-LTE modelling of the He I $10830 \AA$ line in early-type main sequence stars. 2005, Astron. Astrophys., 443, 293, DOI: 10.1051/0004-6361:20053412

Przybilla, N. \& Butler, K., Non-LTE Line Formation for Hydrogen Revisited. 2004, Astrophys. J., 609, 1181, DOI: 10.1086/421316

Przybilla, N., Nieva, M. F., \& Edelmann, H., NLTE Analyses of sdB Stars: Progress and Prospects. 2006, Baltic Astronomy, 15, 107

Sahal-Bréchot, S. \& Segre, E. R. A., Semi-Classical Calculations of Electron and Ion Collisional Broaden- ing of the Strongest U. V. Ionic Lines of Astrophysical Interest. 1971, Astron. Astrophys., 13, 161

Schaffenroth, V. 2015, Ph.D. Thesis, University of Erlangen-Nuremberg 